\title{
Experiencias Teórico-Prácticas de Física en un Entorno Virtual
}

\section{0}

\section{José Daniel Sierra Murillo ${ }^{\mathrm{a}}$}

a Universidad de La Rioja, Departamento de Química, Área de Física Aplicada; Complejo Científico-Tecnológico, C/ Madre de Dios 51 26006-Logroño, daniel.sierra@unirioja.es.

\begin{abstract}
An innovative project on Experimental Theoretical Practices in Physics in a Virtual Environment 3.0 is proposed. The aim is to improve the competences of the student's autonomous and collaborative work. Supported both on a variant of the "Flipped Learning" Methodology and within a Virtual Environment 3.0. Obviously, in addition to the improvement in the learning of Physics, it is of great interest the acquisition by students of computer skills, very important in a scientific-technological environment of the 21st century. The development of the project is carried out in three Phases: Introduction, Preparation and Application of the methodology used to achieve the fundamental objectives of this project. Some of the results pursued by this project are, among others, corroborate the importance of using the new technologies of the information and communication society, not only in the transmission of information, but also in the processes of University learning. All of it, within a Virtual Environment 3.0 or, even, within an approximation to 4.0. This profile of the project combines with the philosophy of Bologna 2020 regarding changes in teaching-learning models.
\end{abstract}

\section{Keywords:}

Theoretical-Practical Experiences, Physics, Competences, Autonomous and Collaborative Work, Flipped Learning Methodology, Informatica Skills, Virtual Environment 3.0

\section{Resumen}

Se propone un proyecto innovador sobre Experiencias Teórico-Prácticas en Física en un Entorno Virtual 3.0. Se pretende la mejora de las competencias del trabajo autónomo y colaborativo del alumno. Apoyadas ambas sobre una variante de la Metodología "Flipped Learning" y dentro de un Entorno Virtual 3.0. Obviamente que además de la mejora en el aprendizaje de la Física, es de gran interés la adquisición por parte del alumnado de habilidades informáticas, muy importantes en un entorno científicotecnológico del siglo XXI. El desarrollo del proyecto se lleva a cabo en tres Fases: Introducción, Preparación y Aplicación de la metodología utilizada para alcanzar los objetivos fundamentales de este proyecto. Alguno de los resultados que se persiguen con este proyecto son, entre otros, corroborar la importancia de la utilización de las nuevas tecnologías de la sociedad de la información y de la comunicación, no solo en la transmisión de información, 
sino también en los procesos de aprendizaje universitario. Todo ello, dentro de un Entorno Virtual 3.0 ó, incluso, dentro de una aproximación al 4.0. Este perfil del proyecto conjuga con la filosofía de Bolonia 2020 relativa a los cambios en los modelos de docencia-aprendizaje.

Palabras clave: Experiencias Teórico-Prácticas, Física, Competencias, Trabajo Autónomo y Colaborativo, Metodología "Flipped Learning", Habilidades Informáticas, Entorno Virtual 3.0.

\section{Introducción}

La educación superior ha experimentado un gran cambio dentro del sistema educativo de la sociedad actual en diferentes ámbitos (Ortega Carrillo, 2004; González Mariño, 2008) como, por ejemplo:

- La evolución de los procesos de formación desde entornos presenciales convencionales hacia otros ámbitos más amplios.

- La demanda generalizada para que los estudiantes adquieran competencias específicas y transversales (Bolonia, 2009), así como ciertas habilidades necesarias para su aprendizaje continuo.

El espacio de aprendizaje ha progresado de forma vertiginosa. Las instituciones educativas, ya sean presenciales o a través de entornos virtuales, tienen que reajustar sus sistemas de intercambio de información y comunicación. Estos cambios traen consigo el desarrollo de nuevas estructuras organizativas de la enseñanza, caracterizadas por la modularidad, la interconexión y la flexibilidad/capacidad de intercambio sobre entornos virtuales diversos.

Esto exige a las instituciones de educación, entre otras la superior, flexibilidad en sus procedimientos y en su estructura administrativa, de acuerdo a las necesidades de una nueva sociedad (Ortega Carrillo, 2004).

Las instituciones actuales deben responder a este desafío, revisar sus referentes actuales y promover experiencias innovadoras en los procesos de enseñanza-aprendizaje. Para ello, pueden apoyarse en las nuevas Tecnologías de la sociedad de la Información y de la Comunicación (TIC), sin olvidar hacer énfasis en la docencia. Cambios de estrategias didácticas de los profesores y alumnos para acceder, buscar, compartir y gestionar toda la información referida a diferentes materiales objeto de estudio y aprendizaje, es decir, en los procesos de innovación docente. (Mora Mora H., Signes Pont, M. T., De Miguel Casado, G. and Gilart Iglesias, V., 2015)

La Universidad dispone de multitud de experiencias de «enseñanza virtual», «aulas virtuales», etc. No obstante, se necesita la participación activa y la motivación del profesorado y alumnado, además de un fuerte compromiso institucional con la innovación

(c)) EY-NC-ND 2017, Universitat Politècnica de València 
docente y su puesta en valor al mismo nivel que la investigación específica de dichos actores, profesorado y alumnos. El devenir actual de la actividad universitaria promueve la investigación, a veces, en detrimento de la docencia y de los proyectos de innovación docente (Salinas, 2004). Cuando estos procesos innovadores docentes pueden ser métodos de mejora de la actividad universitaria, tanto docente como investigadora. Más aún, se olvida a veces en algunas instituciones que la formación básica en los primeros cursos de las diferentes titulaciones es fundamental para un sólido crecimiento del aprendizaje del alumnado en la diversidad de conocimientos necesarios hoy en día. Como competencias específicas y transversales, así como habilidades asociadas, relacionados todos ellos con el afán de mejora profesional de los actores implicados.

En cuanto al Entorno Virtual 3.0 (EV-3.0), se considera como un estado en el que Internet proporciona condiciones de gran interés para que los individuos y las organizaciones puedan compartir información independientemente de las redes y dispositivos que utilicen. (Mora Mora H., Azorín López, J., Jimeno Morenilla, A., Sánchez Romero, J. L., Pujol López, F., García Rodríguez, J., Serra Pérez, J. A., Morell Giménez, V., Rives Pérez, M. F., Saval Calvo, M., García García, A. and Orts Escolano, S., 2016) Las Webs 3.0 también se han venido a denominar Webs semánticas (Berners-Lee, 2001; W3C, 2001). Este nuevo concepto de $W e b$ semántica constituye esencialmente una extensión de la Web tradicional en la que la información se coloca de forma estructurada para permitir su fácil consulta y acceso tanto por interlocutores humanos como por sistemas automáticos (Silva, J. M., Mahfujur, A. S. Md. and El Saddik, A., 2008; Nacer and Aissani, 2014).

En el ámbito educativo esas herramientas adquieren más relevancia debido a que contribuyen a revisar los planteamientos docentes actuales. Los estudiantes, ya no solo tienen que ser buenos conocedores de cada materia en cuestión, sino también deben desarrollar otras actitudes como la creatividad, el espíritu crítico y la capacidad para el aprendizaje continuo que la sociedad les demanda. Todo ello, en línea con la Declaración Mundial para la Educación Superior en el siglo XXI (Granados, 2011) y la Declaración de Bolonia 2020 (Bolonia, 2009).

Seguidamente se describen brevemente los apartados de los que consta este artículo. A continuación (Sección "Objetivos"), se exponen los objetivos que se pretenden alcanzar a través de este proyecto innovador. Posteriormente, y dentro de la Sección "Desarrollo de la innovación", se describe el desarrollo de la metodología utilizada en este proyecto mediante una planificación en tres Fases. Por lo que respecta a la Sección titulada "Resultados", en ella se muestra una comparativa de las mejoras observadas en los mismos. Finalmente, se termina con unas breves conclusiones que confirman el potencial del uso racional de las TIC en el estudio Teórico-Práctico de la Física.

\section{Objetivos}

Los objetivos principales de este proyecto innovador, basado en Experiencias TeóricoPrácticas en Física sobre un EV-3.0, son la mejora de las competencias del trabajo autónomo y colaborativo del alumnado. Para la mejora de éste último, los tradicionales 
Grupos Reducidos (GR $\rightarrow$ entre 25 y 30 alumnos) se subdividen en pequeños subGrupos Reducidos (sbGR $\rightarrow$ entre 3 y 5 alumnos) con el objetivo de un mejor seguimiento (individual y colectivo) por parte del profesor (Mazur, 1997). Dichos objetivos competenciales se consiguen de una forma más eficiente mediante la utilización de la Metodología "Flipped Learning" (MFL), (Prieto, 2015), dentro de un EV-3.0. Aunque es obvio el objetivo de mejora en el aprendizaje de la Física, es de gran interés la adquisición por parte del alumno diversas habilidades informáticas, muy importantes en un entorno científico-tecnológico en pleno siglo XXI. (Karpicke and Blunt, 2011; Deslauriers, L., Schelew, E. and Wieman C., 2011; Freeman, S., Eddy, S. L., McDonough, M., Smith M. K., Okoroafor N., Jordt, H. and Wenderoth, M. P., 2014) En la sección correspondiente a la parte metodológica, se describen los objetivos y, además, se muestra el desarrollo del proyecto llevado a cabo en tres Fases: Introducción, Preparación y Aplicación de la metodología utilizada para alcanzar los objetivos fundamentales de este proyecto. Alguno de los resultados que se persiguen con este proyecto son, entre otros, corroborar la importancia de la utilización de las nuevas tecnologías de la sociedad de la información y de la comunicación, no solo en la transmisión de información, sino también en los procesos de aprendizaje universitario, todo ello, dentro de un EV-3.0. Este perfil del proyecto conjuga con la filosofía de Bolonia 2020 relativas a los cambios en los modelos de docencia-aprendizaje.

\section{Desarrollo de la innovación}

La planificación de la metodología utilizada en este proyecto consta de tres Fases. Pretendería evolucionar la enseñanza tradicional (centrada en el protagonismo del profesor que explica todo a sus alumnos) hacia otro tipo de enseñanza en la que el protagonismo se reparta más entre el profesor y sus alumnos. Para que sean estos los que profundicen en el estudio de los temas objeto de investigación teórico-práctica a partir de los materiales y/o referencias proporcionados previamente por el profesor. Todo ello, a través de un trabajo autónomo-colaborativo dentro de un EV-3.0.

El trabajo autónomo y colaborativo del alumno en la preparación de experiencias teóricoprácticas de Física a través de la MFL y la utilización de un EV-3.0 es sumamente interesante. Por ello, el objetivo fundamental de una de las Fases (Fase 2), es buscar la eficacia autónoma y colaborativa de cada alumno en dicho trabajo de preparación como miembro activo de un sbGR. La optimización de esta Fase 2, con un posible asesoramiento complementario del profesor, promueve una mejora sustancial en el buen desarrollo posterior de la experimentación teórico-práctica.

Sobre todo, porque otra de las Fases en este proyecto (Fase 3) necesita un trabajo constructivo/eficiente de los equipos de alumnos (denominados sbGR) en el desarrollo resolutivo de las mencionadas experiencias teórico-prácticas: análisis de las mismas, propuestas de posibles vías de resolución (una o varias) y comprobación de las mismas, reflejando en el correspondiente documento los razonamientos por los que se ha elegido

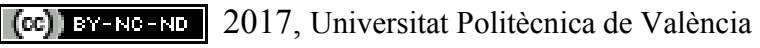


dichas vías de resolución, el proceso de resolución matemático y la selección física de las soluciones matemáticas obtenidas.

Además, la posibilidad de transmitir/compartir la información obtenida/generada en todo el proceso de aprendizaje a través de los mencionados entornos virtuales crea hábitos de transparencia y superación entre los alumnos.

\section{Fase 1: Introducción metodológica.}

Una vez se haya impartido uno de los temas de la asignatura de Física, el profesor propondrá a cada equipo de trabajo (sbGR con 3-5 alumnos) una tipología de experiencias teórico-prácticas para su estudio y preparación mediante una variante de la MFL. Los alumnos, a través del EV-3.0 (sobre todo, con la ayuda del aula virtual Blackboard Learn ${ }^{\mathrm{TM}}$ versión 9.1, que forma parte de este entorno), dispondrán de la información necesaria: número de sbGR al que pertenecen (sbGR.A1.01, el alumno pertenece al subgrupo reducido 01, dentro del Grupo Reducido (GR) A1), la mencionada tipología de ejercicios teóricoprácticos asignada a cada sbGR, documentación correspondiente a la temática vinculada con dicha tipología y ya tratada en clase, con amplia información sobre la misma: referencias bibliográficas en papel y online, etc.; pero podrán complementarla con información obtenida por ellos de forma autónoma pero contrastada con el profesor.

No obstante, en la $1^{\text {a }}$ sesión de experiencias teórico-prácticas, el profesor expondrá/mostrará a los alumnos, dentro del entorno presencial (también posible en el EV3.0) del aula, los siguientes puntos fundamentales para un buen trabajo teórico-práctico:

- El programa de trabajo.

- La forma de trabajo de un científico/tecnólogo en un proceso resolutivo teórico-práctico basado en una variante del Método Científico.

- El material relativo a las experiencias teórico-prácticas utilizado para la realización de cada una de las mencionadas experiencias de Física.

- El material informático (hardware y software) que forma parte del EV-3.0 del que disponen en la universidad.

\section{Fase 2: Preparación metodológica.}

Para comenzar el proceso de preparación del trabajo teórico-práctico (trabajo autónomo y/o colaboración virtual entre los miembros de cada sbGR y el profesor), cada sbGR utilizará toda la información recibida por parte del profesor a través de una vía fundamental del mencionado EV-3.0 (Aula Virtual de la asignatura). También se permite la utilización de otras vías de obtención de información complementarias, pero sujetas a un control científico-tecnológico llevado a cabo por el profesor. A lo largo de esta $2^{\mathrm{a}}$ Fase, cada sbGR podrá consultar dudas al profesor mediante tutorías presenciales y/o virtuales, y también con sus compañeros de sbGR, GR y/o curso. El objetivo fundamental de esta Fase 2 es buscar la efíciencia en el aquí indicado trabajo de preparación de cada sbGR, así como un buen desarrollo posterior de cada experiencia teórico-práctica en la correspondiente sesión en GR. Cada sbGR añadirá en un documento Word, correspondiente a su práctica, toda la 
información (textual, imágenes/audio/video y referencias de todo tipo) obtenida/generada en esta Fase 2.

\section{Fase 3: Aplicación metodológica.}

Una vez trabajada con suficiencia por parte de los alumnos (...con posibilidad de apoyo del profesor...) la Fase 2, previa, y ya dentro del aula en la correspondiente sesión en GR:

a) Cada sbGR comenzará el trabajo resolutivo de una experiencia teórico-práctica vinculada con la tipología que se les haya propuesto, para su realización y finalización al cabo de una hora de cada sesión en GR. Hará uso del material obtenido/generado en el proceso de preparación previo, ya que las observaciones anotadas por los miembros del sbGR a lo largo del proceso de preparación anteriormente indicado (Fase 2) serán de gran ayuda. Las posibles dudas que podrían permanecer insatisfechas, además de otras que sobre el proceso resolutivo definitivo surgieran en el mismo, podrán ser consultadas al profesor, teniéndolas en cuenta en la evaluación de esta Fase 3:

- Es muy importante recordar al alumno la importancia de una completa/correcta preparación sobre la tipología teórico-práctica que se les asignó con la finalidad de evitar posibles errores conceptuales y/o metodológicos.

- Por otra parte, también es conveniente supervisar que el alumno utilice adecuadamente la mencionada información/metodología con el fin de que consolide unas muy útiles habilidades necesarias en su posterior dedicación discente/docente, investigadora $\mathrm{y} / \mathrm{o}$ profesional en general.

b) Una vez terminado el proceso resolutivo, los alumnos de cada sbGR completarán el documento (en el que se introduce toda la información generada en las tres Fases) con una interpretación de los resultados obtenidos, así como de la justificación de la vía/metodología resolutiva elegida para llegar a ellos.

- Conviene recordar al alumno la importancia del correcto uso del Método Científico en el proceso de experimentación teórico-práctica en ciencia/tecnología y objeto de nuestro estudio en Física. La forma de trabajo de un científico/tecnólogo en un proceso resolutivo teórico-práctico debe tener siempre presente la filosofía del "importantísimo" Método Científico.

c) Con toda la información obtenida en el proceso global de Experimentación TeóricoPráctica (textual, imágenes/audio/video y referencias de todo tipo que complementen todo el proceso de aprendizaje), el responsable de cada sbGR enviará al profesor y compartirá con sus compañeros de asignatura esa información a través de la plataforma virtual de la misma.

La posibilidad de transmitir/compartir la información obtenida/generada en todo el proceso de aprendizaje a través de los mencionados entornos virtuales crea hábitos de transparencia y superación entre los alumnos.

En cuanto a la planificación temporal o cronograma del proyecto, cada una de las Experiencias Teórico-Prácticas en Grupos Reducidos (GR) podrá desarrollarse en periodos de dos semanas, a la finalización de cada tema de la asignatura relacionada con la Física.

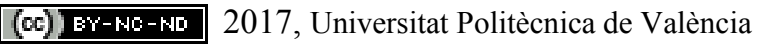


Las horas estimadas de dedicación al proyecto por cada uno de los componentes del grupo de innovación serán en torno a 20 horas, dependiendo de la titulación a la que pertenezca cada Grupo Reducido (GR) de alumnos.

\section{Resultados}

Dentro de diversas muestras de resultados, se ha analizado la mejoría que supone el método aquí presentado respecto al tradicional sobre un mismo tipo de alumnado (estudiantes de Ingeniería Industrial) y a través de dos tipos de medidas. Una subjetiva, relacionada con la satisfacción expresada por el alumnado al profesor a la finalización del trabajo, así como su actitud en el desarrollo del mismo a través de esta metodología. La otra cuantitativa, (ver Fig. 1), con base en la nota obtenida en la $1^{a}$ prueba presencial o convocatoria de febrero. ${ }^{1}$ En ella se aprecia la influencia del Método 3.0 (Con esta denominación se hará referencia a la Metodología "Flipped Learning" dentro de un EV-3.0) sobre los alumnos a lo largo del semestre que dura el desarrollo del trabajo teórico-práctico poniéndose de manifiesto el compromiso que adquieren y el reto de asumir presentarse casi la totalidad de los alumnos matriculados a dicha $1^{\text {a }}$ convocatoria $(98 \%)$, cuando antes lo hacía el $65 \%$. Y sobra decir que con mucho mejores resultados: Superan la $1^{\text {a }}$ convocatoria $41 \%$ (Método 3.0 ) por $18 \%$ (Método tradicional).

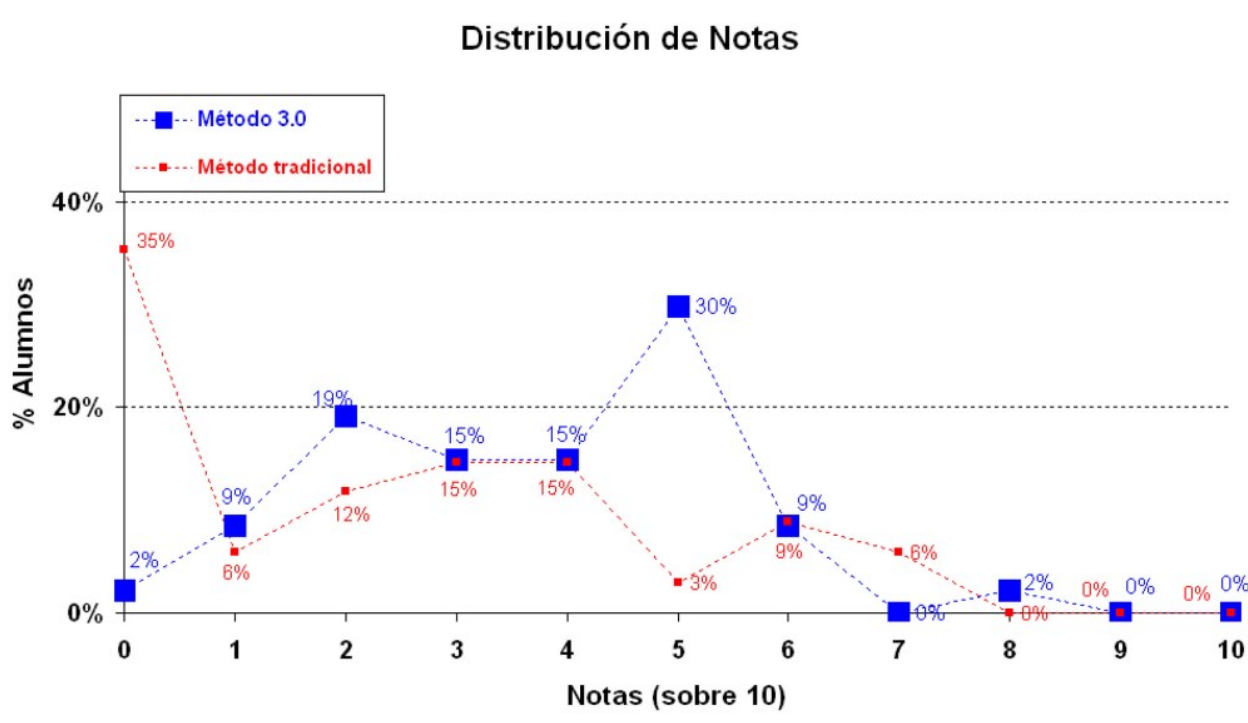

Figura 1. Comparativa de dos modelos utilizados antes (tradicional) y ahora (Método 3.0).

\footnotetext{
1 Es importante tener en cuenta que el \% de alumnos se refiere a la Tasa de Rendimiento. Se calcula sobre el número de alumnos matriculados. Sobra decir que el rendimiento llega a niveles razonables una vez agotadas el resto de convocatorias,
} 
Dentro de los resultados obtenidos a lo largo de la puesta en práctica del proyecto de innovación aquí presentado, se exponen a continuación los puntos más significativos observados a lo largo del periodo utilizado (semestre):

* Se produce una evidente mejoría del trabajo autónomo y colaborativo entre los alumnos de cada sbGR, y también entre alumnos-profesor, principalmente a través del EV-3.0.

Además, es significativo el progreso del alumnado en la utilización de:

- La conexión entre plataformas informáticas (hardware y software) con sistemas experimentales donde la adquisición de datos se realiza de forma automática.

- Las plataformas informáticas (hardware y software) para el análisis de las resoluciones teórico-prácticas por parte del alumno.

- Ciertos entornos virtuales utilizados para la transmitir y compartir la información utilizada y generada en todo el proceso por los mismos alumnos y entre alumnosprofesor.

* Pero, sobre todo, existe una muy apreciable mejora cualitativa y cuantitativa en el proceso de aprendizaje Teórico-Práctico en Física por parte de los alumnos, así como de sus resultados académicos. (Fig. 1)

Me gustaría remarcar que los resultados académicos obtenidos mediante esta variante de la MFL son más realistas pues la nota máxima (10) no existe en las calificaciones obtenidas por los alumnos. Con ello, se intenta recordar a los alumnos relacionados con el mundo científico-tecnológico que "a la perfección se intenta tender...", a través de una adecuada utilización de Método Científico.

\section{Conclusiones}

Las conclusiones que pueden extraerse de los resultados obtenidos a lo largo del desarrollo de este proyecto de innovación docente confirman un potencial real de la utilización racional de las TIC y la MFL. En un proceso sólido y fiable de aprendizaje TeóricoPráctico de la Física de los Sistemas Naturales y Artificiales (I+D+i), y con la inestimable ayuda de un EV-3.0.

Una óptima preparación previa del trabajo teórico-práctico que se va a desarrollar en el GR mediante esta variante de la MFL, así como la puesta en común de este proceso a través del mencionado EV-3.0 hacen de dicha metodología una herramienta muy interesante para obtener una mejora, tanto en el aprendizaje consolidado de la Física como los resultados académicos.

Igualmente, el intercambio a través del EV-3.0 de las resoluciones teórico-prácticas, el análisis de las mismas, los resultados obtenidos a partir de ellos y el debate constructivo entre alumnos y entre alumnos-profesor proporcionan un rico caldo de cultivo que alimenta un crecimiento científico-tecnológico sólido y respetuoso con el medio ambiente.

(c) EY-NC-ND 2017, Universitat Politècnica de València 
Sobra decir que todo el proceso está embebido en una praxis en torno a las TIC, que mejora las habilidades del alumnado respecto a todo aquello relacionado con esta nueva realidad virtual que ya está instalada entre nosotros.

\section{Referencias}

BERNERS-LEE, T., HENDLER, J. y LASSILA, O. (2001). The Semantic Web: A new form of Web content that is meaningful to computers will unleash a revolution of new possibilities. Scientific American, Vol. 284: pp. 35-43.

BOLONIA. (2009). The Bologna Process 2020 - The European Higher Education Area in the new decade. Communiqué of the Conference of European Ministers Responsible for Higher Education.

DESLAURIERS, L., SCHELEW, E. y WIEMAN C.. (2011). Improved Learning in a LargeEnrollment Physics Class, Science, Vol. 332, pp. 862-864. DOI: 10.1126/science.1201783.

FREEMAN, S., EDDY, S. L., MCDONOUGH, M., SMITH M. K., OKOROAFOR N., JORDT, H. y WENDEROTH, M. P. (2014). Active learning increases student performance in science, engineering, and mathematics, Proc. Natl. Acad. Sci. USA, Vol. 111, pp. 8410-8415.

GONZÁLEZ MARIÑO, J. C. (2008). TIC y la transformación de la práctica educativa en el contexto de las sociedades del conocimiento. Revista de Universidad y Sociedad del Conocimiento (RUSC). Vol. 5, n. ${ }^{\circ} 2$.

GRANADOS, J. (2011). The Challenges of Higher Education in the 21st Century, GUNi Newsletter, 5/11. (http://www.guninetwork.org/articles/challenges-higher-education-21st-century).

KARPICKE, J. D. y BLUNT, J. R. (2011). Retrieval Practice Produces More Learning than Elaborative Studying with Concept Mapping, Science, Vol. 331, pp. 772-775. DOI: 10.1126/science. 1199327.

MAZUR, E. (1997). Peer Instruction: A User's Manual. New York: Prentice Hall Series in Educational Innovation.

MORA MORA H., AZORÍN LÓPEZ, J., JIMENO MORENILLA, A., SÁNCHEZ ROMERO, J. L., PUJOL LÓPEZ, F., GARCÍA RODRÍGUEZ, J., SERRA PÉREZ, J. A., MORELL GIMÉNEZ, V., RIVES PÉREZ, M. F., SAVAL CALVO, M., GARCÍA GARCÍA, A. y ORTS ESCOLANO, S. (2016). Nuevas tendencias web 3.0 para la mejora de los procesos docencia-aprendizaje. Innovaciones metodológicas en docencia universitaria: resultados de investigación (pp.1543-1558). Alicante: Universidad de Alicante, Vicerrectorado de Estudios, Formación y Calidad, Instituto de Ciencias de la Educación.

MORA MORA H., SIGNES PONT, M. T., DE MIGUEL CASADO, G. y GILART IGLESIAS, V. (2015). Management of social networks in the educational process, Computers in Human Behavior, Vol. 51, Part B, pp. 890-895. doi:10.1016/j.chb.2014.11.010.

NACER, H. y AISSANI, D. (2014). Semantic web services: Standards, applications, challenges and solutions, Journal of Network and Computer Applications, Vol. 44, pp. 134-151. DOI: 10.1016/j.jnca.2014.04.015. 
ORTEGA CARRILLO, J. A.. (2004). Redes de aprendizaje y curriculum intercultural. Actas del XIII Congreso Nacional y II Iberoamericano de Pedagogía. Ed. Sociedad Española de Pedagogía. Valencia.

PRIETO MARTÍN, A. (2015-12-21). Decálogo de innovación metodológica para que los alumnos aprendan más y mejor en las asignaturas universitarias. Blog Profesor 3.0. http://profesor3punto0.blogspot.com.es/2015/12/decalogo-de-innovacion-metodologica.html.

SALINAS, J. (2004). Innovación docente y uso de las TIC en la enseñanza universitaria. Revista Universidad y Sociedad del Conocimiento. Vol. $1, \mathrm{n} .^{\mathrm{o}} 1$.

SILVA, J. M., RAHMAN, A. S. y EL SADDIK, A. (2008). Web 3.0: a vision for bridging the gap between real and virtual. Paper presented at the 1st ACM international workshop on Communicability design and evaluation in cultural and ecological multimedia system, Vancouver British Columbia, Canada.

W3C. (2001). W3C Semantic Web Activity. URL: http://www.w3.org/2001/sw/, Acceso: mayo 2016. 